\title{
Segmented crystalline scintillators: Empirical and theoretical investigation of a high quantum efficiency EPID based on an initial engineering prototype CsI(TI) detector
}

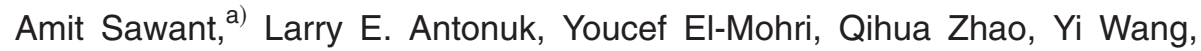 \\ Yixin Li, and Hong Du \\ Department of Radiation Oncology, University of Michigan, Ann Arbor, Michigan 48103
}

Louis Perna

Saint Gobain Crystals, Newbury, Ohio 44065

(Received 7 December 2005; revised 12 January 2006; accepted for publication 27 January 2006; published 28 March 2006)

\begin{abstract}
Modern-day radiotherapy relies on highly sophisticated forms of image guidance in order to implement increasingly conformal treatment plans and achieve precise dose delivery. One of the most important goals of such image guidance is to delineate the clinical target volume from surrounding normal tissue during patient setup and dose delivery, thereby avoiding dependence on surrogates such as bony landmarks. In order to achieve this goal, it is necessary to integrate highly efficient imaging technology, capable of resolving soft-tissue contrast at very low doses, within the treatment setup. In this paper we report on the development of one such modality, which comprises a nonoptimized, prototype electronic portal imaging device (EPID) based on a $40 \mathrm{~mm}$ thick, segmented crystalline $\mathrm{CsI}(\mathrm{Tl})$ detector incorporated into an indirect-detection active matrix flat panel imager (AMFPI). The segmented detector consists of a matrix of $160 \times 160$ optically isolated, crystalline $\mathrm{CsI}(\mathrm{Tl})$ elements spaced at $1016 \mu \mathrm{m}$ pitch. The detector was coupled to an indirect detection-based active matrix array having a pixel pitch of $508 \mu \mathrm{m}$, with each detector element registered to 2 $\times 2$ array pixels. The performance of the prototype imager was evaluated under very low-dose radiotherapy conditions and compared to that of a conventional megavoltage AMFPI based on a Lanex Fast-B phosphor screen. Detailed quantitative measurements were performed in order to determine the $x$-ray sensitivity, modulation transfer function, noise power spectrum, and detective quantum efficiency (DQE). In addition, images of a contrast-detail phantom and an anthropomorphic head phantom were also acquired. The prototype imager exhibited approximately 22 times higher zero-frequency DQE $(\sim 22 \%)$ compared to that of the conventional AMFPI $(\sim 1 \%)$. The measured zero-frequency DQE was found to be lower than theoretical upper limits $(\sim 27 \%)$ calculated from Monte Carlo simulations, which were based solely on the x-ray energy absorbed in the detector-indicating the presence of optical Swank noise. Moreover, due to the nonoptimized nature of this prototype, the spatial resolution was observed to be significantly lower than theoretical expectations. Nevertheless, due to its high quantum efficiency $(\sim 55 \%)$, the prototype imager exhibited significantly higher DQE than that of the conventional AMFPI across all spatial frequencies. In addition, the frequency-dependent DQE was observed to be relatively invariant with respect to the amount of incident radiation, indicating x-ray quantum limited behavior. Images of the contrast-detail phantom and the head phantom obtained using the prototype system exhibit good visualization of relatively large, low-contrast features, and appear significantly less noisy compared to similar images from a conventional AMFPI. Finally, Monte Carlo-based theoretical calculations indicate that, with proper optimization, further, significant improvements in the DQE performance of such imagers could be achieved. It is strongly anticipated that the realization of optimized versions of such very high-DQE EPIDs would enable megavoltage projection imaging at very low doses, and tomographic imaging from a "beam's eye view" at clinically acceptable doses. (C) 2006 American Association of Physicists in Medicine. [DOI: 10.1118/1.2178452]
\end{abstract}

Key words: portal imaging, MTF, DQE, high quantum efficiency, segmented crystals, imageguided radiotherapy

\section{INTRODUCTION}

Recent advances such as three-dimensional (3D) conformal radiotherapy and intensity-modulated radiotherapy have made it possible to better achieve the central goal of radiotherapy-delivering maximum dose to the tumor vol- ume while sparing normal tissue and critical organs, through the use of significantly reduced treatment margins and steeper dose gradients. The successful realization of such techniques has been greatly facilitated by increasingly sophisticated forms of image guidance for target localization and geometric verification of dose delivery. ${ }^{1}$ A significant 
amount of this effort has been directed toward imaging the tumor volume in the treatment room itself. ${ }^{2-11}$ Such effort is largely motivated by a strong desire within the community to reduce dependence on surrogates such as bony landmarks, thereby avoiding errors in dose delivery due to organ motion. ${ }^{12}$ One such strategy is to acquire diagnostic-quality tomographic images with the patient in the treatment position. Typically, this arrangement involves a kilovoltage $(\mathrm{kV})$ $\mathrm{x}$-ray tube and an active matrix flat panel imager (AMFPI) mounted on the linear accelerator (linac) gantry, both oriented orthogonally to the beam axis. ${ }^{6,7,13}$ Such $\mathrm{kV}$ imaging systems integrated into the linac are becoming of increasing clinical interest due to their ability to provide high-quality images with soft-tissue contrast at relatively low doses $(\sim 2 \mathrm{cGy}){ }^{8}$

A parallel approach to that described above is to make use of the treatment beam itself to obtain megavoltage computed tomographic (MVCT) images. ${ }^{14,15}$ In this regard, numerous strategies employing AMFPI ${ }^{16-21}$ and non-AMFPI (Refs. 22-26) technologies have been reported. Although anatomical structures have inherently lower contrast at MV energies compared to $\mathrm{kV}$, it has been demonstrated by various groups that soft-tissue contrast is nonetheless achievable through MVCT using conventional as well as investigational electronic portal imaging devices (EPIDs). ${ }^{8,16,20,25}$ In particular, obtaining MVCT images that are reconstructed from "beam's eye view" projection data using AMFPI EPIDs (which have become a standard add-on to modern linacs) is of tremendous interest since it eliminates the need for additional equipment and minimizes geometric uncertainties that can possibly occur in an orthogonally mounted $\mathrm{kV}$ imaging system. In addition, megavoltage CT numbers can potentially yield more accurate information for radiotherapy dose calculations and inhomogeneity corrections..$^{14,15,27,28}$ Moreover, MVCT images are less subject to x-ray scatter (due to the fact that Compton scatter at MV energies is largely forward directed $^{29}$ ) and are less affected by the presence of metal objects such as dental implants, hip prostheses, etc., present in the imaged volume-factors that can cause significant artifacts in kVCT images. ${ }^{16,30-33}$

The primary hurdle that prevents megavoltage cone-beam CT from being clinically feasible is the relatively low x-ray quantum efficiency (QE) of the detectors used in current AMFPI EPIDs. ${ }^{8}$ As a result of this low QE, the dose required to achieve a reasonable level of soft-tissue delineation is prohibitively high and, thus, clinically impractical. ${ }^{8,20}$ Recent studies have demonstrated that MVCT images exhibiting soft-tissue contrast can be obtained at greatly reduced doses that are comparable to those used for diagnostic CT through the use of high-QE detectors. ${ }^{16-18,25}$ In addition, the incorporation of high-QE detectors in EPIDs is also likely to greatly benefit projection MV, i.e., portal, imaging. For example, such imagers would enable the acquisition of high-quality portal images at a fraction of a centigray (cGy), enabling more frequent imaging in order to reduce set-up errors. Furthermore, obtaining a continuous sequence of such highquality, low-dose images during treatment opens up the pos- sibility of various forms of image-guided adaptive radiotherapy. It should be noted that, alternative strategies to achieve dose reduction in megavoltage tomographic and projection imaging, using relatively low-QE detectors, are also being investigated. ${ }^{16,18,34}$ The incorporation of high-QE detectors, coupled with such strategies, is likely to result in further improvement in the overall imaging performance. ${ }^{16,34}$

In a recent study, we reported on the theoretical performance of high-QE, segmented crystalline scintillator-based EPIDs. ${ }^{35}$ Previous empirical investigations of megavoltage imagers based on such thick, segmented crystalline detectors have been reported by Mosleh-Shirazi et al., using a TVcamera-based EPID (Refs. 23 and 36) and, more recently, by Seppi et al., using an AMFPI EPID. ${ }^{17}$ However, due to limitations in fabrication techniques, such detectors were subject to a significant tradeoff between size and uniformity of the element pitch (i.e., center-to-center spacing of the elements) and, therefore, could only be fabricated so as to have regularly spaced elements with a relatively coarse pitch $(\sim 3000 \mu \mathrm{m}) \quad$ (Ref. 36) or elements with a finer pitch $(\sim 380 \mu \mathrm{m})$ but significantly nonuniform center-to-center spacing. ${ }^{17}$ Ongoing improvements in engineering and fabrication technology are helping to progressively mitigate this tradeoff. Recently, such improvements have enabled the fabrication of an initial, nonoptimized version of a $40 \mathrm{~mm}$ thick, segmented CsI(Tl) detector with a $1016 \mu \mathrm{m}$ element pitch. In this work we report empirical and theoretical results on the performance of a megavoltage imager based on this "engineering test prototype," coupled to an indirect-detection active matrix flat panel array. Measurements of x-ray sensitivity, modulation transfer function (MTF), noise power spectrum (NPS), and the detective quantum efficiency (DQE) are reported at $6 \mathrm{MV}$. Images of a contrast-detail phantom and a head phantom are shown along with similar images acquired using a conventional AMFPI. Measurements are compared with theoretical upper limits obtained from Monte Carlo calculations for an optimized version of the prototype detector. ${ }^{35}$ Finally, the implications of the realization of such very high-DQE EPIDs for MVCT are discussed.

\section{METHODS AND MATERIALS}

\section{A. Prototype EPID design}

Current megavoltage EPIDs use phosphor screen-based detectors which face a severe tradeoff between x-ray quantum efficiency and spatial resolution due to the spread of optical photons, which in turn limits the DQE. A segmented detector circumvents this tradeoff by ensuring that the light generated within a cell as a result of $\mathrm{x}$-ray interactions remains confined within that cell. ${ }^{37}$ Based on various design considerations, described in a previous publication, ${ }^{35}$ a prototype, nonoptimized, $16 \times 16 \times 4 \mathrm{~cm}^{3} \mathrm{CsI}(\mathrm{Tl})$ segmented detector was fabricated at St. Gobain Crystals and Detectors, $\mathrm{OH}$.

The detector [Fig. 1(a)] consisted of 16 small-area ( $\sim 4$ $\times 4 \mathrm{~cm}^{2}$ ) single-crystal blocks, which were obtained from a single $\mathrm{CsI}(\mathrm{Tl})$ ingot in order to ensure that all of the blocks 


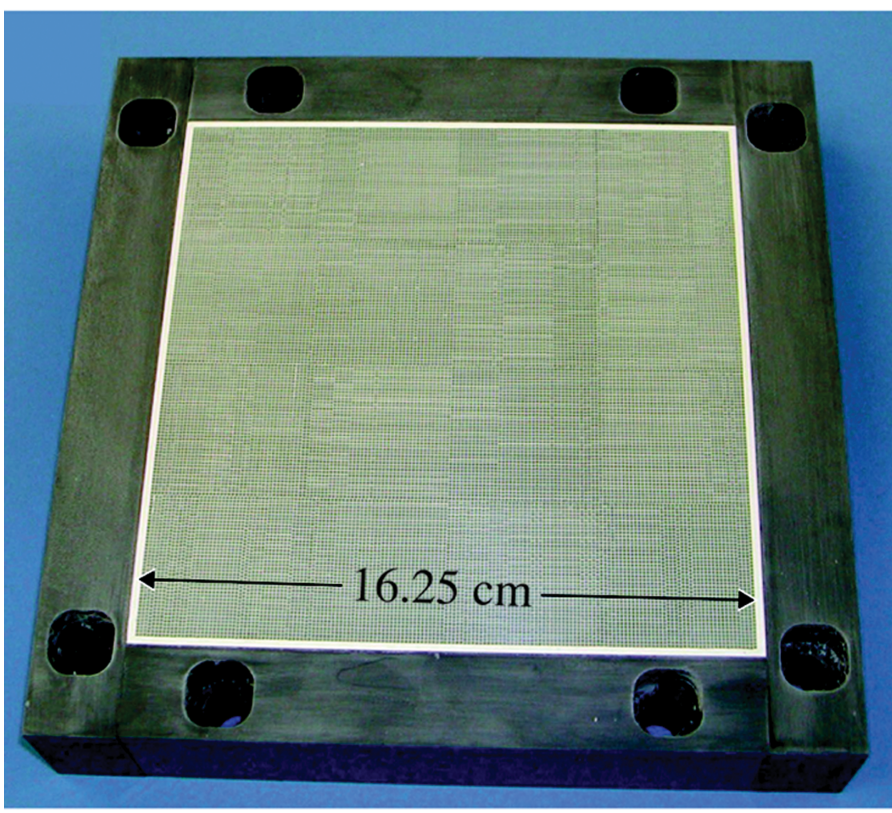

(a)

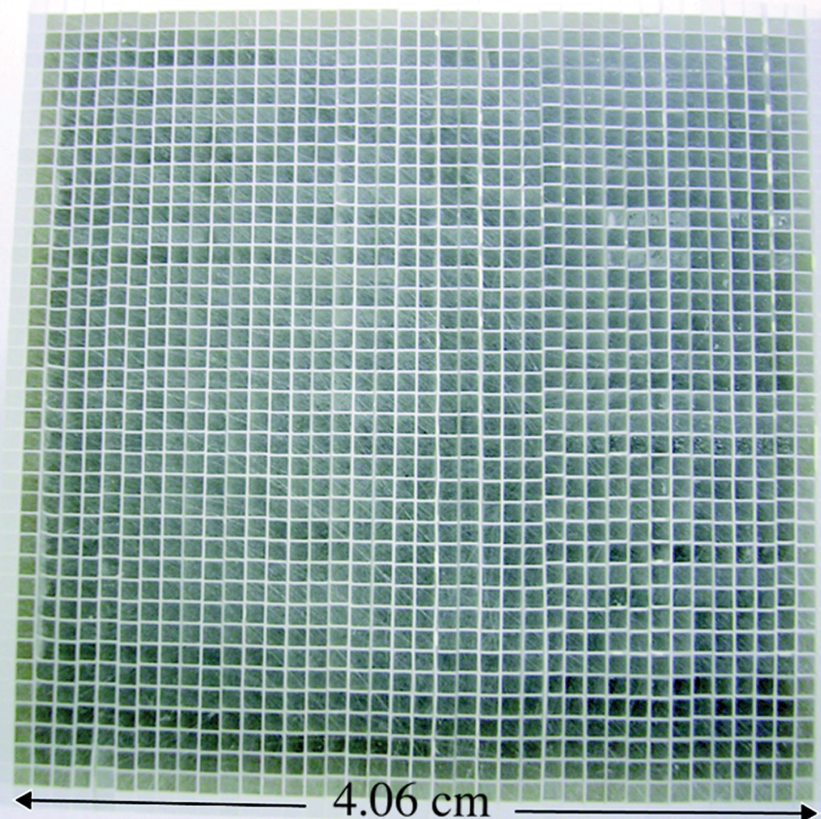

(b)

Fig. 1. (a) Prototype segmented $\mathrm{CsI}(\mathrm{Tl})$ detector. The detector matrix is composed of a $4 \times 4$ array of smaller submatrices which are precisely aligned to each other under a microscope. The bars attached to the sides of the detector matrix are made of PVC in order to minimize x-ray scatter, and are used to mount the detector on the alignment jig. (b) Magnified view of a submatrix. It can be seen that in this early prototype, the elements exhibit highly regular center-to-center spacing in one direction (i.e., between columns) while the spacing in the orthogonal direction (i.e., between rows) shows small nonuniformities.

had identical physical properties. Using high-precision machining techniques, each small-area block was diced at regularly spaced intervals $(1016 \mu \mathrm{m})$, first along the $X$ and, subsequently, along the $Y$ dimension, to form a two-dimensional matrix of $40 \mathrm{~mm}$ tall $\mathrm{CsI}(\mathrm{Tl})$ elements separated by $100 \mu \mathrm{m}$ thick, $40 \mathrm{~mm}$ deep gaps. An optically opaque and reflective polymer was introduced within the gaps in order to create a submatrix of $40 \times 40$ optically isolated $\mathrm{CsI}(\mathrm{Tl})$ elements with a center-to-center spacing of $1016 \mu \mathrm{m}$ [Fig. 1(b)]. With this technique, the individual $\mathrm{CsI}(\mathrm{Tl})$ elements exhibited highly regular center-to-center spacing along one direction [i.e., between the columns in Fig. 1(b)], while the spacing in the orthogonal direction was slightly nonuniform. Sixteen such submatrices were arranged such that the well-aligned elements of all sub-matrices were oriented in the same direction, and precisely aligned under a microscope in order to form a larger-area $\left(16.25 \times 16.25 \mathrm{~cm}^{2}\right)$ segmented detector matrix shown in Fig. 1(a). The top (x-ray side) and bottom (array side) surfaces of the entire detector were slightly roughened by polishing them with fine-grain sand paper in order to make them optically diffuse. Such surface treatment helps to reduce optical glare that may arise due to multiple reflections of light rays between interfaces (e.g., the detector and the array). ${ }^{38}$

The segmented $\mathrm{CsI}(\mathrm{Tl})$ detector was coupled to an indirect detection active matrix array (508 $\mu \mathrm{m}$ pitch), previously developed for radiotherapy applications. ${ }^{39}$ The detector and the array were housed in a custom-built, precision alignment jig (Fig. 2), which allowed horizontal, vertical, and rotational adjustment of the $\mathrm{CsI}(\mathrm{Tl})$ matrix with respect to the underly- ing array. The jig design incorporated spring-loaded bolts under the array in order to provide upward pressure so as to ensure good optical coupling with the segmented detector. The detector was aligned with respect to the active matrix array such that the columns of the detector (Fig. 1) were perpendicular to the direction of the gate lines of the array and the $\mathrm{CsI}(\mathrm{Tl})$ elements were registered to $2 \times 2$ underlying array pixels. Registration was performed through iterative positioning of the $\mathrm{CsI}(\mathrm{Tl})$ matrix with respect to the array.

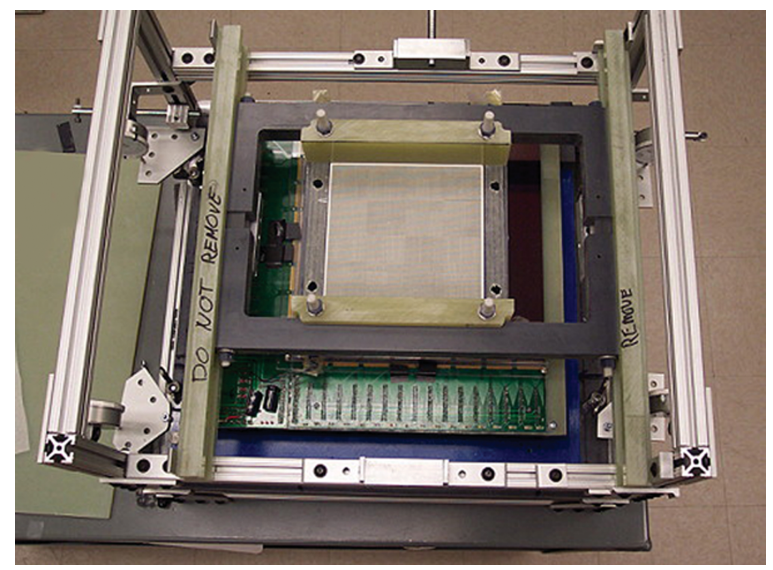

FIG. 2. Segmented detector+active matrix array placed in a precision alignment jig which enables horizontal, vertical, and rotational adjustment of the $\mathrm{CsI}(\mathrm{Tl})$ matrix with respect to the underlying array. The outer framework of the jig is composed of $\mathrm{Al}$ in order to provide adequate strength and rigidity, while the inner framework is composed of polymer-based materials in order to minimize the effect of $\mathrm{x}$-ray scatter from the jig. 
For each iteration, the registration was verified under the $\mathrm{x}$-ray beam in the following manner. An open-field image was acquired corresponding to a given alignment and the degree of variation between adjacent array pixels along each gate and data line was recorded. A smaller amount of variation in signal values indicated that the scintillator part of the detector elements was overlying the array photodiodes. In contrast, the appearance of a band-like pattern in the image indicated that one or more septal walls were overlying the array photodiodes. The alignment corresponding to the least signal variation along each line was chosen as the configuration representing the best element-to-array registration. As expected, the registration was observed to be slightly better along the gate line direction compared to the data line direction.

\section{B. Measurements}

The signal and noise performance of the prototype EPID was examined under radiotherapy imaging conditions (6 MV photon beam from a Varian linear accelerator). A $\sim 1.2 \mathrm{~mm}$ thick steel plate, similar to the $1 \mathrm{~mm}$ thick $\mathrm{Cu}$ plate used in conventional AMFPI EPIDs, ${ }^{40}$ was placed on top in order to provide buildup and filtration of low-energy scattered radiation. One side of the steel plate was polished to a "mirrorlike" finish. Measurements were performed using three different optical layers on the top (i.e., x-ray side) of the CsI(Tl) detector-a diffuse or lambertian reflective layer formed by a white sheet of photo-quality paper placed between the steel plate and the $\mathrm{CsI}(\mathrm{Tl})$ detector, a specular reflective layer, formed by coupling the mirrorlike surface of the steel plate directly to the detector, and an optically absorptive layer formed by a black paper placed between the steel plate and the detector. (For convenience, we shall refer to these three layers as "white," "mirror," and "black," respectively.) Measurements of signal, noise, and spatial resolution were performed for each of the three configurations as a function of the irradiation time of the linac in monitor units (MU). [For the calibration used on this linac, $1 \mathrm{MU}$ corresponds to 0.8 cGy deposited in water at a source-to-detector distance (SDD) equal to $100 \mathrm{~cm}$, with $10 \mathrm{~cm}$ overlying water, for a field size of $10 \times 10 \mathrm{~cm}^{2}$ at the linac isocenter (i.e., sourceto-axis distance $=100 \mathrm{~cm})]$. The data obtained from these measurements were used to determine the x-ray sensitivity, NPS and MTF of the prototype imager. For the aforementioned signal and noise measurements, the AMFPI was operated in fluoroscopic mode and the radiation was delivered at a rate of $100 \mathrm{MU} / \mathrm{min}$, while the MTF measurements were performed in radiographic mode, at $600 \mathrm{MU} / \mathrm{min}$. The fluoroscopic mode of operation involves continuous image frame acquisition in synchronization with the beam pulses from the linac. In the radiographic mode, a single image frame is acquired after the linac delivers a programmed amount of radiation. Further details of the fluoroscopic and radiographic operation of this array may be found elsewhere. ${ }^{41-43}$ In each case, appropriate offset and gain corrections were applied to individual image frames and the corrected pixel data in each frame were binned in groups of $2 \times 2$ pixels in order to match the pixel pitch of the active matrix array $(508 \mu \mathrm{m})$ to that of the segmented detector elements $(1016 \mu \mathrm{m})$. In addition, measurements were also performed using a Lanex Fast-B phosphor screen $\left(\sim 133 \mathrm{mg} / \mathrm{cm}^{2} \mathrm{Gd}_{2} \mathrm{O}_{2} \mathrm{~S}: \mathrm{Tb}\right.$, Eastman Kodak, Rochester, NY) $+1 \mathrm{~mm} \mathrm{Cu}$ plate coupled to the same active matrix array as that used for the prototype EPID. The Fast-B-based data were also binned in groups of 2 $\times 2$ pixels and were processed in a manner similar to that used for the data acquired from the prototype imager, in order to facilitate direct comparisons.

\section{X-ray sensitivity}

The signal response of the prototype AMFPI was measured as a function of $\mathrm{x}$-ray irradiation. The imager was placed at a SDD of $130 \mathrm{~cm}$, and a field size of $10 \times 10 \mathrm{~cm}^{2}$ at the isocenter was used. Image frames were acquired in synchronization with the beam pulses. Frame acquisition was synchronized with the beam pulses using the "Target I" output from the linac control logic. The acquisition system, previously developed for research involving such active matrix arrays, ${ }^{44}$ allowed the reading of up to 100 gate lines of the array between consecutive beam pulses delivered at $100 \mathrm{MU} / \mathrm{min}$ (corresponding to a pulse frequency of $60 \mathrm{~Hz}$ ). For images corresponding to multiple beam pulses, the system software was programmed so as to trigger the data acquisition after the delivery of the desired number of pulses. (Note that the actual frame readout in this case still occurred between beam pulses.) The number of monitor units per pulse, approximately 0.027 , was determined by counting the number of pulses from the "Target I" output for a programmed MU setting. For each data sequence, 800 dark and 800 flood data frames (i.e., frames in the absence and presence of radiation, respectively) were acquired. For each data set, the average of the 800 dark frames was subtracted from the average of the 800 flood frames to yield the signal. The signal values were converted from analog-to-digital converter $(A D C)$ units to electrons using a measured calibration factor of $1 \mathrm{ADC} \approx 7480 e$. (The calibration was performed through a separate measurement involving the injection of a known amount of charge into the acquisition system preamplifiers and recording the corresponding ADC values.) The slope of the X-ray signal response plotted as a function of the irradiation time yielded the $\mathrm{x}$-ray sensitivity in units of e/MU.

\section{Modulation transfer function (MTF)}

MTF for the prototype detector was measured, using the angled slit technique ${ }^{45}$ - a method widely employed for characterizing the spatial resolution of $\mathrm{kV}$ as well as MV digital imaging systems. ${ }^{37,43,46-48}$ Two tungsten blocks with precision machined and polished opposing faces, having dimensions of $19 \times 8.5 \times 4.25 \mathrm{~cm}^{3}$, were separated by $0.01 \mathrm{~cm}$ shims to form a long, narrow slit of dimensions $19 \times 8.5$ $\times 0.01 \mathrm{~cm}^{3}$. The longest dimension of the blocks $(19 \mathrm{~cm})$ was positioned along the beam central axis, so as to constitute the "thickness" of the slit. The slit was centered with respect to the radiation source in order to maximize the sig- 
nal, and positioned such that the exit surface of the slit was almost in contact with the detector, which was placed at a SDD of $138 \mathrm{~cm}$. In addition, the slit was oriented at an angle of $\sim 3^{\circ}$ with respect to the data line direction of the active matrix array [i.e., the direction parallel to the "columns" of the detector as seen in Fig. 1(a)]. The field size was adjusted to $6.5 \times 6.5 \mathrm{~cm}^{2}$ at the exit surface of the slit. Image frames were acquired by operating the imager in radiographic mode. A total of ten images, each at $0.5 \mathrm{MU}$, were acquired. Owing to the fact that the $\mathrm{x}$-ray source of the linac gives a divergent beam, it is possible that the small amount of radiation that penetrates through the tungsten blocks creates a non-uniform radiation profile that is superimposed on the slit images. Such an effect can potentially cause distortion in the baseline of the line spread function (LSF) calculated from these images. In order to minimize this effect, images of the radiation profile were acquired by displacing the slit $\sim 0.5 \mathrm{~cm}$ away from the center of the beam. Gain and offset corrections were applied to all image data sets and the radiation profile images were subtracted from the slit images. For each prototype detector, the final, corrected images were averaged, binned $(2 \times 2)$, and used to estimate the LSF. The absolute value of the Fourier transform of the LSF yielded the onedimensional MTF.

\section{Noise power spectrum}

Dark and flood image frames acquired for the sensitivity measurements described above, were used to estimate noise power spectra (NPS) of the system. The NPS was determined as a function of spatial frequency for irradiation times of 0.027 and $0.054 \mathrm{MU}$ - corresponding to one and two beam pulses, respectively, from the linac. Offset and gain corrections were applied to each flood frame. A selective $3 \times 3$ median filter was applied in order to correct for defective array pixels, affecting less than $0.2 \%$ of the total number of pixels per frame. The pixel ADC values were converted into units of electrons and the image frames were binned as described in the previous section. One-dimensional noise power spectra were determined from these images using the synthesized slit technique, which is described in detail elsewhere, ${ }^{43,49}$ and is briefly summarized as follows. From the acquired data, and after binning, 800 independent, nonoverlapping blocks (i.e., slits), each with $90 \times 45$ pixels, were selected such that the longer dimension of the blocks was parallel to the gate lines of the array. Each slit was summed along the narrow direction to form a 90-point realization. Low frequency background trends were subtracted and a Hanning window was applied to each realization. A 1D Fourier transform was applied to each of the 800 realizations and the resulting power spectra, were appropriately normalized and averaged to yield a 1D NPS. The NPS data were subsequently corrected in order to compensate for the noisereduction effect caused by frame-to-frame charge carry-over, i.e., lag. (First frame lag was determined through independent measurements to be $\sim 5 \%$ under radiotherapy conditions.) Details of the correction technique may be found elsewhere. ${ }^{50,51}$

\section{Detective quantum efficiency (DQE)}

The measured MTF and NPS (with lag correction) were used to determine the frequency-dependent DQE for the prototype imager. DQE was calculated using the relation

$$
\operatorname{DQE}(u)=\frac{A^{2} T_{\mathrm{sys}}^{2}(u)}{q_{0} S_{\mathrm{sys}}(u)},
$$

where $u$ represents the independent spatial frequency variable along one axis, $A$ is the average signal per binned pixel (obtained from the flood frames used to calculate the NPS), $q_{0}$ is the incident x-ray fluence, obtained from published data, ${ }^{52,53}$ while $T_{\text {sys }}(u)$ and $S_{\text {sys }}(u)$ represent the 1D MTF and NPS of the system, respectively.

\section{Lubberts effect}

As detector thickness increases, there occurs a correspondingly higher degree of variation in the shape of the point spread function (PSF) observed at the output, for signal generated at various depths within an X-ray detector. Due to this variation, the observed MTF of the entire detector is proportional to the weighted sum of the MTFs at each depth, while the observed NPS is proportional to the sum of the squares of these individual MTFs. As a result of this dissimilar integration across the various "layers" of the detector, $T_{\text {sys }}^{2}(u)$ falls off faster with respect to spatial frequency than $S_{\text {sys }}(u)$, thereby contributing to a falloff in DQE values at higher spatial frequencies [Eq. (1)]. This phenomenon was first investigated by Lubberts ${ }^{54}$ and has thereafter been referred to as the Lubberts effect.

In the case of megavoltage imaging detectors based on conventional powdered phosphor screens that are a few hundred microns thick, energy deposition is relatively uniform as a function of screen thickness. Therefore, the Lubberts effect occurs primarily due to the depth-dependent nature of optical transport within the phosphor screen. In the case of much thicker detectors such as the segmented $\mathrm{CsI}(\mathrm{Tl})$ prototype, there is significant variation in energy deposition as well as in the optical transport along the thickness dimension (i.e., depth). Thus, for such detectors, DQE degradation induced by the Lubberts effect occurs due to the depth dependence of energy deposition as well as the depth dependence of the optical transport.

The degree of DQE degradation due to the Lubberts effect can be quantified by calculating a factor $\mathrm{R}(u)$, previously defined by Nishikawa et al. ${ }^{55}$ and hereafter referred to as the Lubberts fraction. This factor is determined from the MTF and the NPS as

$$
R(u)=\frac{T_{\text {sys }}^{2}(u)}{S_{\text {profile }}(u)},
$$

where $S_{\text {profile }}(u)$ gives the shape of the system NPS 


$$
S_{\text {profile }}(u)=\frac{S_{\mathrm{sys}}(u)}{S_{\mathrm{sys}}(0)} .
$$

In this study, the Lubberts fraction for the prototype imager was calculated as a function of spatial frequency from the empirically determined MTF and NPS.

\section{Monte Carlo simulations}

The inherent signal, spatial resolution and noise properties of the prototype detector were examined through Monte Carlo simulations of the x-ray energy absorbed in the detector. The results of these simulations were used to calculate the intrinsic MTF, NPS, and DQE. For a given detector, these values represent theoretical upper limits of performance of any imaging system incorporating that detector. ${ }^{35}$ (Note that, in order to completely characterize the performance of a segmented detector, it is also necessary to perform detailed modeling of the optical transport within the scintillator elements. However, such optical modeling is beyond the scope of the present study.) The methodology used for these calculations has been explained in detail in an earlier publication, ${ }^{35}$ and is briefly summarized as follows. An idealized version of the prototype detector (coupled to a $1 \mathrm{~mm}$ thick $\mathrm{Cu}$ plate), which assumed perfect cell-to-cell alignment between the detector elements, was defined within the DOSXYZNRC Monte Carlo user code. ${ }^{56}$ A 6 MV photon spectrum corresponding to that from a Varian linac was used. ${ }^{53}$ The one-dimensional (1D) MTF and NPS corresponding to the energy absorbed within the detector were determined by simulating the angled slit technique (to estimate the LSF) and the synthesized slit technique, respectively, and the DQE was calculated from Eq. (1). In addition, the Lubberts fraction corresponding to the depth-dependent energy deposition within the prototype detector was calculated from Eq. (2), using the Monte Carlo-based MTF and NPS.

\section{Images}

In order to qualitatively assess the performance of the prototype EPID, a contrast detail phantom and a anthropomorphic head phantom were imaged at very low doses. Images were acquired fluoroscopically, at $130 \mathrm{~cm}$ SDD with a field size of $15 \times 15 \mathrm{~cm}^{2}$ at the isocenter. As is the case for the signal and noise measurements described above, the two phantoms were also imaged using a Lanex Fast-B phosphor screen $+1 \mathrm{~mm} \mathrm{Cu}$ plate coupled to the active matrix array, and the Fast-B images were binned $(2 \times 2)$ in order to match the effective pixel pitch $(1016 \mu \mathrm{m})$ of the prototype imager.

\section{Contrast-detail phantom}

The phantom consisted of an $8 \mathrm{~mm}$ thick, aluminum slab with three rows of holes of diameters $1.3,0.8$, and $0.5 \mathrm{~cm}$. The holes in each row had depths ranging from 4.6 to $0.3 \mathrm{~mm}$, corresponding to progressively decreasing contrast levels from 1.91 to $0.12 \%$, which were determined as follows. The inherent contrast of each hole with respect to the background was calculated through Monte Carlo simula- tions using the BEAMNRC user code. ${ }^{56,57}$ The geometry consisted of a pencil beam (20 million histories), derived from the same $6 \mathrm{MV}$ photon spectrum as that used in the Monte Carlo calculations described above, incident on $100 \mathrm{~mm}$ diameter slabs of varying thicknesses of aluminum corresponding to the background $(8 \mathrm{~mm})$ and the holes in the contrastdetail phantom. The contrast $C$ for each hole was calculated from

$$
C=200 \frac{\left(I_{2}-I_{1}\right)}{\left(I_{2}+I_{1}\right)} \%,
$$

where $I_{2}$ and $I_{1}$ represent the total number of photons at the exit surface of the slabs corresponding to the hole and the background, respectively. It should be noted that, in a previous publication by our group, ${ }^{39}$ the inherent contrast was calculated for a similar phantom using Eq. (4), considering solely the attenuation coefficient of $\mathrm{Al}$ with respect to a $2 \mathrm{MeV}$ monoenergetic photon beam. These earlier values therefore indicate upper limits of the contrast while the present values derived from the Monte Carlo simulations represent more refined estimates, which account for the photon energy spectrum, the effect of transmitted primary photons, as well as Compton scattered photons and low-energy brehmsstrahlung, all of which can potentially contribute to the image formation process in megavoltage imaging.

Images of the contrast-detail phantom were acquired with the prototype detector with the white top layer. Images were obtained using one and three beam pulses, corresponding to 0.027 and $0.081 \mathrm{MU}$, respectively. Image processing consisted of offset and gain corrections, filtering of nonfunctional pixels, and appropriate window and level adjustments so as to best display the contrast.

\section{Head phantom}

The head phantom (Model 76-018DT, Nuclear Associates, Long Island, NY) consisted of a human skull encased in a tissue-equivalent polymer material. Images were acquired with the prototype detector incorporating a white top layer and a $1 \mathrm{~mm}$ thick $\mathrm{Cu}$ build-up plate [similar to that used in most commercially available AMFPI EPIDs (Refs. 40 and 58)]. The phantom was imaged using two beam pulses (for a total of $0.054 \mathrm{MU}$ ). Image processing similar to that employed for the contrast-detail phantom images was used.

\section{RESULTS}

\section{A. X-ray sensitivity}

Figure 3 shows the signal response for three configurations of the prototype imager consisting of the segmented $\mathrm{CsI}(\mathrm{Tl})$ detector coupled with a white, mirror, and black top layer on the x-ray entrance side. The signal is reported in units of electrons as a function of the irradiation time in MU. For comparison, the signal from a conventional megavoltage imager, based on a Lanex Fast-B screen, is also shown.

It can be seen that the signal response of the prototype imager is highly linear for all three configurations. The configuration with the black top layer exhibits only slightly 


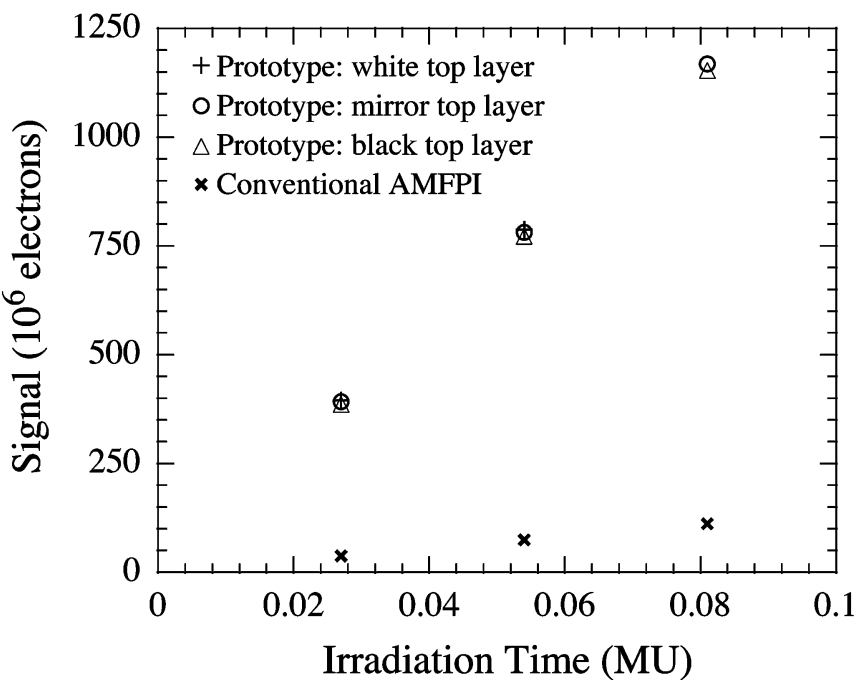

FIG. 3. Signal response of the three different configurations of the segmented detector-based prototype imager as a function of irradiation time in monitor units. The signal value corresponding to $0.081 \mathrm{MU}$ for the configuration with the white top layer was not measured. For comparison, the signal response from a conventional EPID design based on a Lanex-Fast-B phosphor screen, acquired under similar measurement conditions is also shown.

lower x-ray sensitivity $\left(14255 \times 10^{6} \mathrm{e} / \mathrm{MU}\right)$ compared to the configurations using the mirror $\left(14438 \times 10^{6} \mathrm{e} / \mathrm{MU}\right)$ and the white $\left(14592 \times 10^{6} \mathrm{e} / \mathrm{MU}\right)$ top layers, indicating that the nature of the optical properties of the top layer does not significantly affect the signal response.

Such relatively small differences in the sensitivity values for the three configurations suggest that a significant fraction of the light photons generated near the top of the scintillator element is absorbed within the element. This high degree of optical attenuation can be attributed to the high aspect-ratio (i.e., the ratio of element height to cross-sectional area) of the $\mathrm{CsI}(\mathrm{Tl})$ elements, along with the non-ideal reflective and transmissive properties of the septal walls and the scintillator, respectively. Under such conditions, light photons generated near the top of the element are likely to undergo a large number of interactions with the septal walls, resulting in a correspondingly higher degree of absorption within the walls, compared to the light photons generated nearer to the bottom of the element. In addition, due to the greater number of reflections undergone by the light photons generated near the top, these photons traverse a longer optical path in order to reach the bottom surface of the scintillator element. This effect results in further signal reduction due to increased absorption within the scintillator material.

Nonetheless, in all three configurations, the prototype imager exhibits over ten times higher $\mathrm{x}$-ray sensitivity than that measured from the conventional imager $\left(1372 \times 10^{6} \mathrm{e} / \mathrm{MU}\right)$. Such high sensitivity is a result of the significantly higher $\mathrm{x}$-ray quantum efficiency of the prototype detector, estimated from Monte Carlo calculations ${ }^{59}$ to be $\sim 55 \%$ for a $6 \mathrm{MV}$ photon spectrum, ${ }^{53}$ compared to that of a conventional phosphor screen-based detector, which exhibits a quantum efficiency of $\sim 2 \%{ }^{43}$

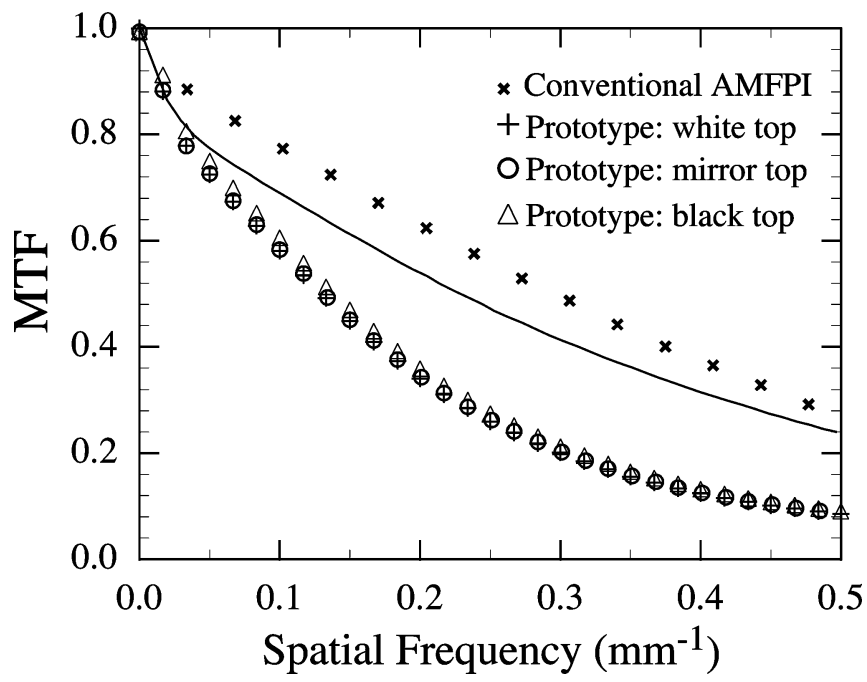

FIG. 4. Presampled MTF for three different configurations of the prototype imager. The solid line represents the upper limit of the MTF for this configuration, calculated from Monte Carlo simulations. For comparison, the measured MTF from a conventional AMFPI, based on a Lanex Fast-B screen and a $1016 \mu \mathrm{m}$ pixel pitch, is also shown.

\section{B. MTF}

Figure 4 shows the spatial resolution of the prototype imager as quantified by the one-dimensional presampled MTF. The plotted points represent the measured MTF for the three configurations of the prototype imager, while the solid line indicates the theoretical maximum MTF estimated from Monte Carlo simulations (Sec. II C). For comparison, the MTF measured from a conventional imager using a Lanex Fast-B screen, normalized to a pixel size of $1016 \mu \mathrm{m}$, is also shown. While the MTF of the prototype detector with the black, optically absorptive, top layer appears to be slightly higher than that of the other two configurations, overall, there is little variation in the spatial resolution with respect to the top layer. Thus, the optical properties of the top layer do not exhibit any significant effect on the resolution. This result appears to be consistent with the relatively low variation in $x$-ray sensitivity with respect to changes in the reflectivity of the top layer. In all three cases, at high spatial frequencies, the measured MTF is significantly lower than the theoretical maximum. This difference is likely due to two resolutiondegrading mechanisms that are present in this early, nonoptimized prototype - non-ideal optical isolation between the scintillator elements, which causes optical crosstalk, and the slight misalignment of the $\mathrm{CsI}(\mathrm{Tl})$ elements along one direction [see Fig. 1(b)], which causes misregistration of the elements with the array pixels. Finally, it is interesting to note that the Monte Carlo-based calculations suggest that an imager using a properly optimized version of the prototype detector could exhibit spatial resolution that is dependent solely on the lateral spread of the absorbed energy and would be only slightly lower than that of a phosphor screen-based system-while gaining over an order of magnitude increase in $\mathrm{x}$-ray quantum efficiency. 

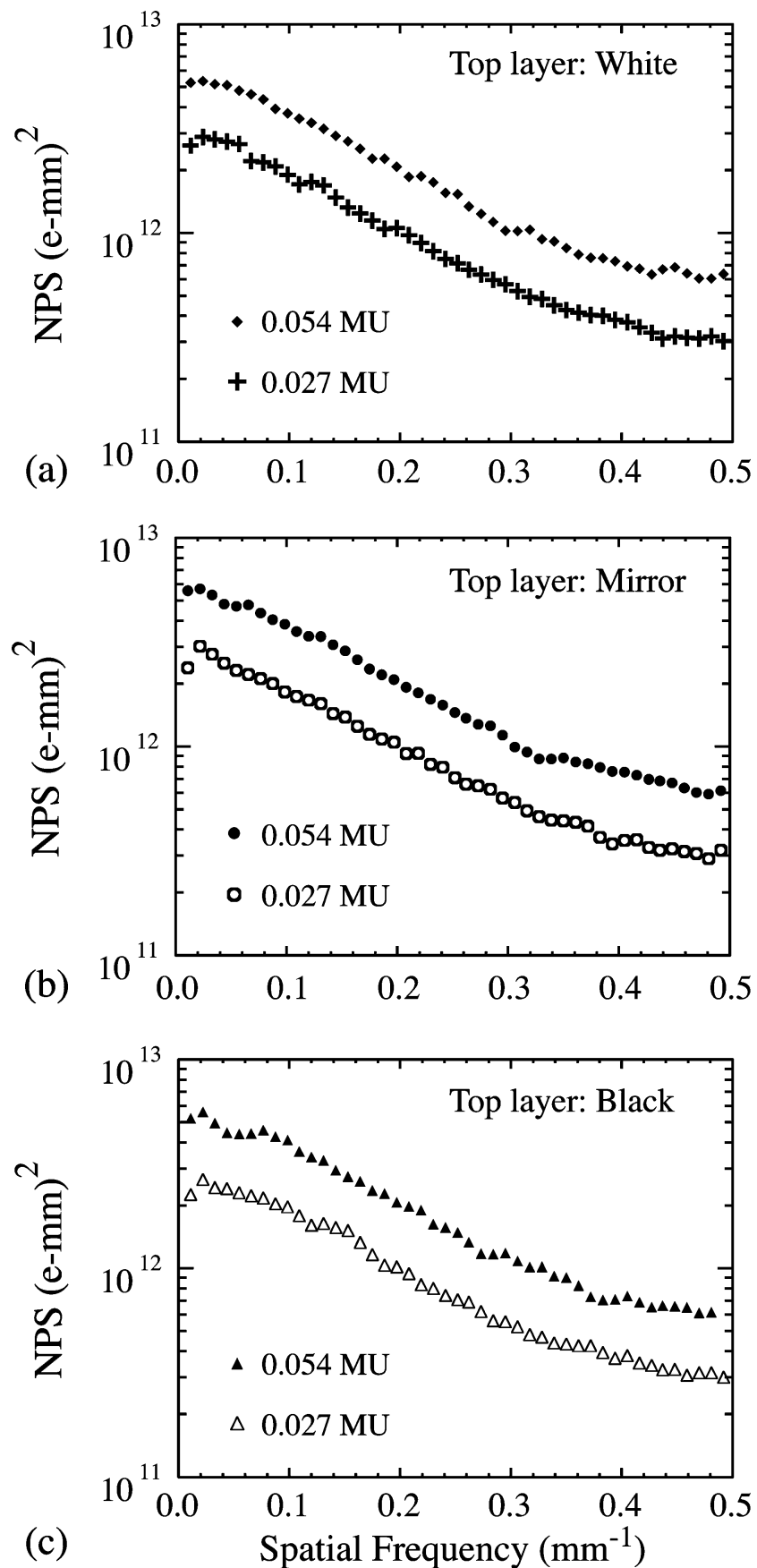

FIG. 5. Noise power spectra measured from the prototype imager with a (a) white, (b) mirror, and (c) black top layer. For each configuration, NPS data are shown at 0.027 and $0.054 \mathrm{MU}$, corresponding to 1 and 2 linac pulses, respectively.

\section{NPS}

Figure 5 shows noise power spectra (corrected for lag) for the prototype imager with the three different optical top layers. Spectra are shown at doses corresponding to one and two beam pulses. The NPS data scale in proportion to dose and exhibit a significant fall-off at higher spatial frequencies.

\section{DQE}

DQE values, calculated from the measured MTF and NPS, are shown in Fig. 6. The solid line represents a theo-

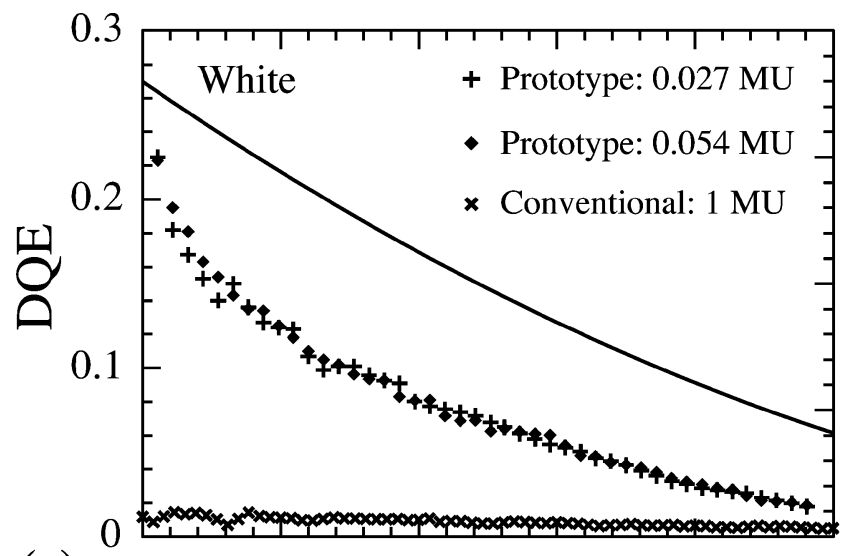

(a) $\begin{array}{llllll}0 & 0.1 & 0.2 & 0.3 & 0.4 & 0.5\end{array}$

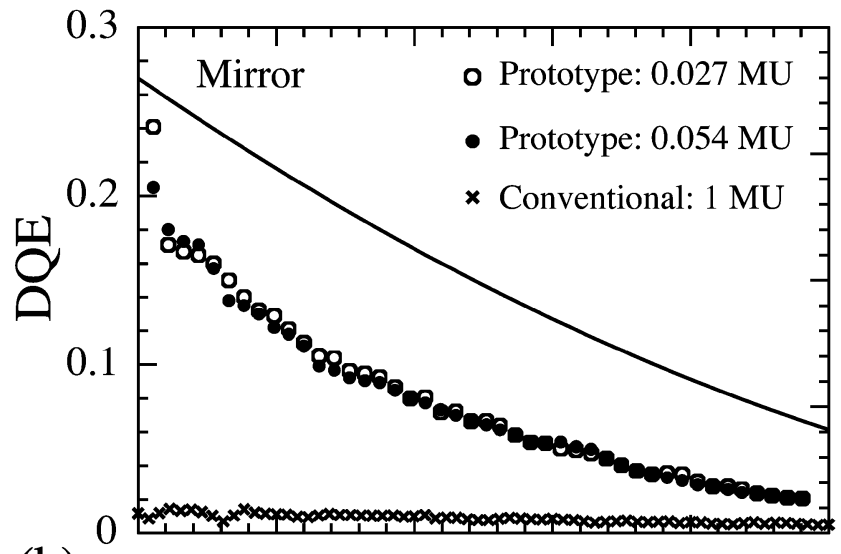

(b)
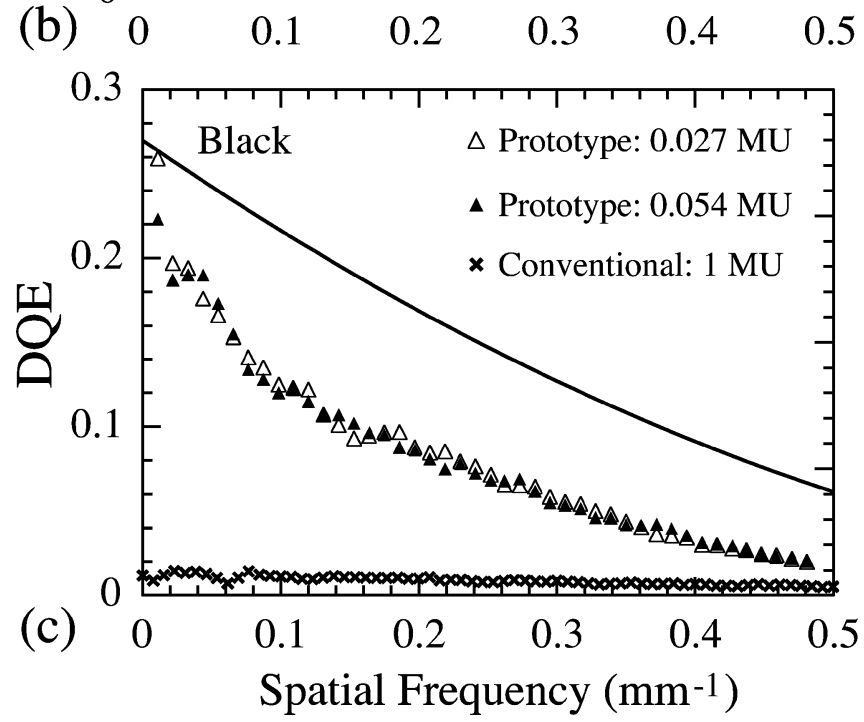

FIG. 6. Detective quantum efficiency of the prototype imager corresponding to a (a) white, (b) mirror, and (c) black top layer. For each configuration, the measured DQE using irradiations of 0.027 and $0.054 \mathrm{MU}$, is shown. The solid line indicates the theoretical maximum values of DQE calculated from Monte Carlo simulations. For comparison, previously published DQE values (Ref. 41) measured from a conventional Lanex Fast-B phosphor screenbased AMFPI (with a $508 \mu \mathrm{m}$ pitch) at $6 \mathrm{MV}, 1 \mathrm{MU}$, are also shown.

retical upper limit for the DQE, calculated from Monte Carlo simulations (Sec. II C). For comparison, previously published DQE values for a conventional Lanex Fast-B phosphor screen-based AMFPI (with a $508 \mu \mathrm{m}$ pixel pitch) under radiotherapy conditions ( $6 \mathrm{MV}, 1 \mathrm{MU})$, are also shown. ${ }^{43}$ In 
all three configurations, the prototype imager exhibits very high DQE, approaching $\sim 22 \%$ at zero spatial frequency-to our knowledge, the highest measured DQE thus far reported for an AMFPI EPID. As is the case for the sensitivity and the MTF measurements, the empirically determined DQE values do not appear to exhibit significant dependence on the optical properties of the top layer. Of particular note is the fact that the DQE is practically independent of the dose, indicating that, even at such low doses, the system is x-ray quantum limited. ${ }^{47}$

An important consideration in the design of high-aspectratio segmented detectors is that light photons generated on the $\mathrm{x}$-ray side of the detector are likely to undergo significantly more reflection and absorption before reaching the pixel photodiode, compared to those generated nearer to the active matrix array-as indicated by the relatively small changes in $\mathrm{x}$-ray sensitivity with respect to changes in the optical reflectivity of the top layer. This effect gives rise to a depth-dependent optical gain and, consequently, increased optical Swank noise, ${ }^{60}$ which can significantly degrade the DQE. $^{37}$ (Swank noise is quantified by the Swank factor which ranges from zero to unity. High Swank factor indicates low noise and vice versa.) It can be seen that measured DQE values near zero spatial frequency for the prototype imager are lower than the calculated theoretical upper limit $(\sim 27 \%)$, the latter of which is based solely on the x-ray energy absorbed within the detector. This difference between the theoretical and measured DQE values near zero frequency is indicative of the presence of optical Swank noise. This is deduced from the fact that the zero frequency DQE can be considered to be the product of the x-ray quantum efficiency of the detector, the Swank factor corresponding to the noise in the absorbed energy distribution (both of which are accounted for in the theoretical DQE calculation) and, the Swank factor corresponding to the noise in the optical pulse distribution. ${ }^{60,61}$ Thus, the optical Swank factor for the prototype can be estimated to be $\sim 0.8$. The presence of nonnegligible but relatively small amount of optical Swank noise suggests that the $\mathrm{CsI}(\mathrm{Tl})$ elements of the prototype detector do exhibit some depth dependence in the optical gain.

The measured DQE exhibits a sharp falloff at higher spatial frequencies, exhibiting significantly lower values than those predicted by the Monte Carlo-based calculation. Nonetheless, due to the much higher x-ray quantum efficiency of the segmented $\mathrm{CsI}(\mathrm{Tl})$ detector compared to that of a Lanex Fast-B phosphor screen, the prototype imager exhibits significantly higher DQE performance than the conventional system across all spatial frequencies. Finally, the theoretical calculation indicates that, for a properly optimized version of the current detector design, even further DQE improvements may be achievable.

\section{E. Lubberts fraction}

Figure 7 shows the empirically and theoretically determined Lubberts fraction, as a function of spatial frequency, for the prototype imager. (Empirical results are shown for the

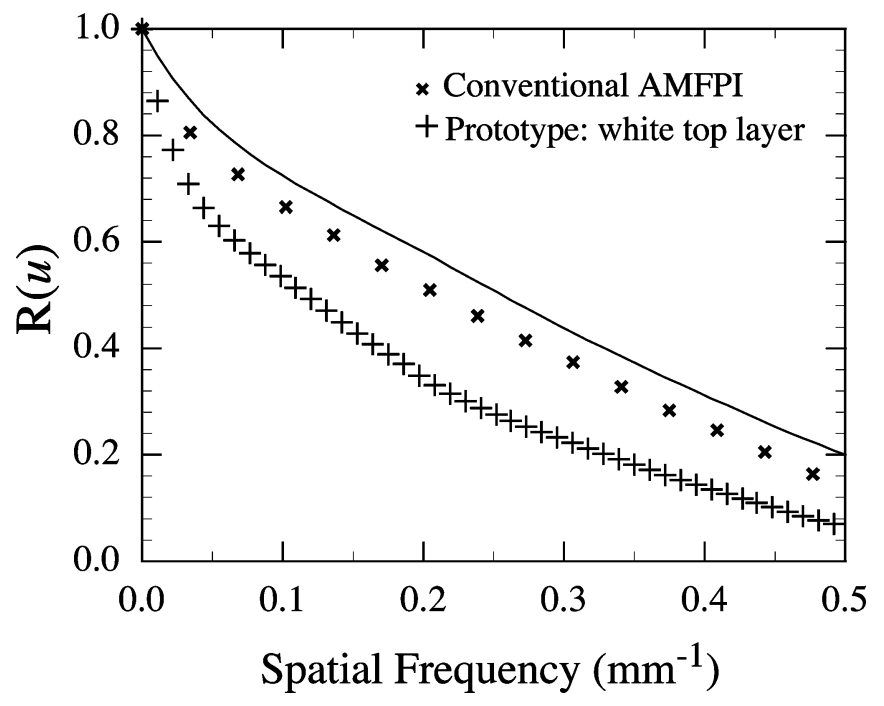

FIG. 7. Lubberts fraction, R(u), calculated from MTF and NPS, using Eq. (2). $\mathrm{R}(u)$ is shown for the prototype imager (incorporating the white top layer) calculated from the measured NPS at $0.027 \mathrm{MU}$ and the measured MTF (+ symbols). For comparison, the empirically determined Lubberts fraction (also at 0.027 MU) for a conventional AMFPI based on a Lanex Fast-B screen and a pixel pitch of $1016 \mu \mathrm{m}$, is also shown ( $\times$ symbols). The solid line represents an upper limit for $\mathrm{R}(u)$ for the segmented detector and is determined from Monte Carlo-based calculations of the MTF and NPS corresponding to the energy absorbed within the detector.

configuration using the white top layer, at $0.027 \mathrm{MU}$. The results from the other two configurations are similar and are not shown for clarity.) Also shown for comparison is the empirically determined Lubberts fraction (also at $0.027 \mathrm{MU}$ ) from a conventional megavoltage AMFPI $(1016 \mu \mathrm{m}$ pixel pitch) based on a Lanex Fast-B screen.

The empirically determined Lubberts fraction for the prototype imager is observed to be lower than that observed for the conventional imager, suggesting that the DQE response of the prototype undergoes significantly more degradation at higher spatial frequencies due to Lubberts effect. As discussed in Sec. II B 5, such degradation occurs due to depthdependent variations in the PSFs (and therefore, in the MTFs) of the absorbed energy as well as the optical quanta. Interestingly, the theoretically calculated Lubberts fraction for the prototype imager indicates that the falloff in DQE due to Lubberts effect corresponding to the absorbed energy is comparable or less than the DQE fall-off occurring in a conventional phosphor screen-based megavoltage imager, the latter of which is due, largely, to optical effects.

These results are indicative of the presence of imperfect optical isolation between adjacent elements of the segmented detector, which in turn is responsible for a significant amount of DQE falloff at higher spatial frequencies. This can be deduced as follows. The optical PSF is determined by the degree of lateral spread of the optical photons. If the septal walls were perfectly opaque, the lateral spreading of these photons into adjacent elements would be prevented. Due to the fact that $\mathrm{CsI}(\mathrm{Tl})$ exhibits relatively high transmissivity, with an optical attenuation length ranging from 100 to $1000 \mathrm{~mm},{ }^{62}$ it is reasonable to assume that the lateral 


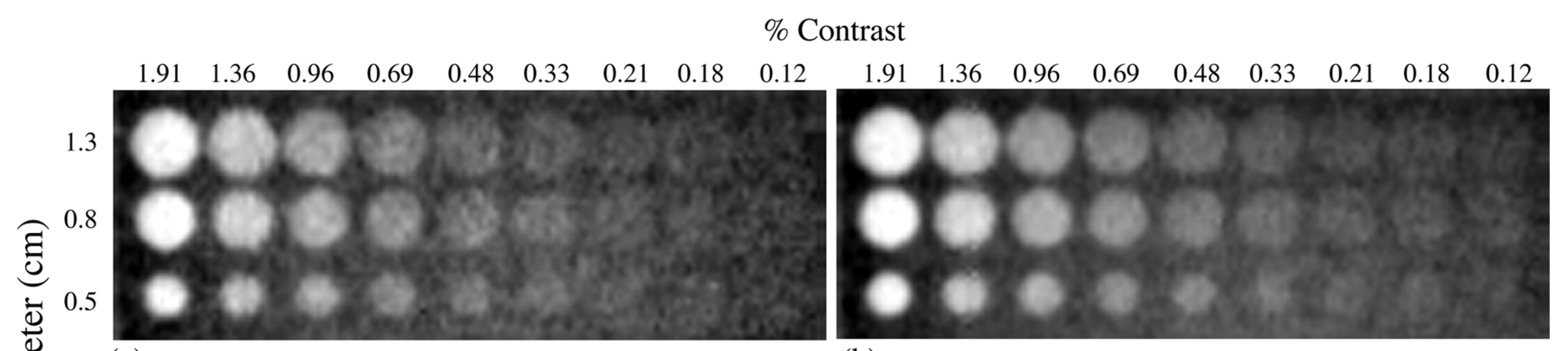

(a)

(b)

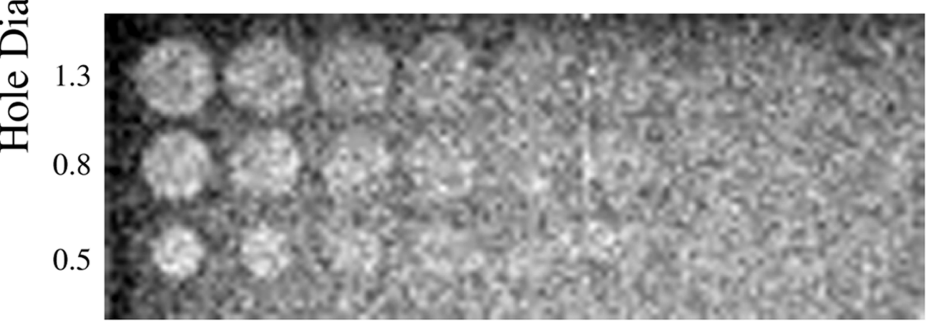

(c)

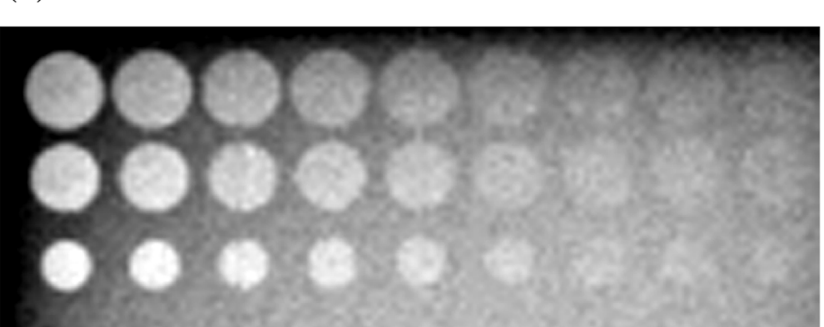

(d)

FIG. 8. Images of a contrast-detail phantom acquired using the prototype imager (incorporating the white top layer) at (a) $0.027 \mathrm{MU}$ and (b) $0.081 \mathrm{MU}$, corresponding to 1 and 3 linac pulses, respectively. The phantom consists of an $8 \mathrm{~mm}$ thick, aluminum slab with three rows of holes, having diameters of (top to bottom) 1.3, 0.8, and $0.5 \mathrm{~cm}$ and hole depths ranging from (left to right) 4.6 to $0.3 \mathrm{~mm}$. The numerical values shown above (a) and (b) represent the inherent contrast (see Sec. II D 1) corresponding to each hole depth. For comparison, images of the same phantom acquired using a conventional AMFPI EPID based on a Lanex Fast-B screen at (c) 0.027 MU and (d) $1 \mathrm{MU}$, are also shown.

spread of the light photons within an element (having a pitch of $1.016 \mathrm{~mm}$ ) will be sufficiently large so that the optical PSF will be equal to the two-dimensional aperture function (i.e., the $2 \mathrm{D}$ rect function) corresponding to the $X-Y$ dimensions of the detector element. Note that this effect does not depend on the depth at which the photons are generated. In other words, due to complete confinement of the light photons generated within an element, the shape of the optical PSF at any depth will be solely determined by the element aperture. In the absence of any depth-dependent variations in the optical PSFs and, provided that there are no significant sources of uncorrelated noise (a reasonable assumption for systems such as the prototype imager, which exhibits very high optical gain and no secondary quantum sink), the optical component of the Lubberts fraction would be unity. In such a situation, DQE degradation due to Lubberts effect would occur solely due to the depth-dependent PSF variations in the absorbed energy and thus, the theoretically and empirically determined values of the Lubberts fraction would be equal. The difference observed in these values (Fig. 7) therefore suggests that a non-negligible fraction of optical photons generated in one element manages to cross over to adjacent elements. Consequently, the optical PSF exhibits significant depth dependence, which ultimately results in a DQE falloff at higher spatial frequencies.

It should be noted that the results presented above, which were performed using a $1.2 \mathrm{~mm}$ thick steel build-up plate, closely match (within $\sim 1.5 \%$ ) the x-ray sensitivity, MTF and DQE values obtained with a $1 \mathrm{~mm}$ thick $\mathrm{Cu}$ build-up plate and white and black top layers. (The results with the $\mathrm{Cu}$ plate are not shown for the sake of conciseness.) The steel plate was used for the results reported in this study because the mirror top layer could not be implemented using copper.

\section{F. Images}

The superior DQE enables the prototype imager to achieve very good contrast resolution (for relatively large objects) at extremely low doses. This can be seen from Figs. 8(a) and 8(b), which show images of the contrast detail phantom acquired using one (0.027 MU) and three (0.081 MU) linac pulses respectively. For comparison, Figs. 8(c) and 8(d) show images of the same phantom acquired under similar conditions, using a conventional Lanex Fast-B screen-based system at doses corresponding to one and 36 linac pulses (0.027 and $1 \mathrm{MU})$, respectively.

The boundaries of the holes appear slightly blurred in the case of images acquired using the prototype system-a result likely due to the relatively poor spatial resolution of the prototype. Nevertheless, at the lowest dose, the image acquired using the prototype system [Fig. 7(a)] appears less noisy and exhibits significantly superior visualization of low-contrast objects compared to the corresponding image acquired using the conventional system [Fig. 7(c)]. Finally, using only $0.081 \mathrm{MU}$, the prototype imager achieves contrast resolution comparable to that achieved by the conventional system at one MU (which corresponds to $\sim 12$ times higher dose than that used by the prototype system).

Figure 9 shows images of a humanoid head phantom acquired using the prototype and a conventional system at $0.054 \mathrm{MU}$. The image obtained from the prototype is less noisy and appears to better resolve low-contrast features 


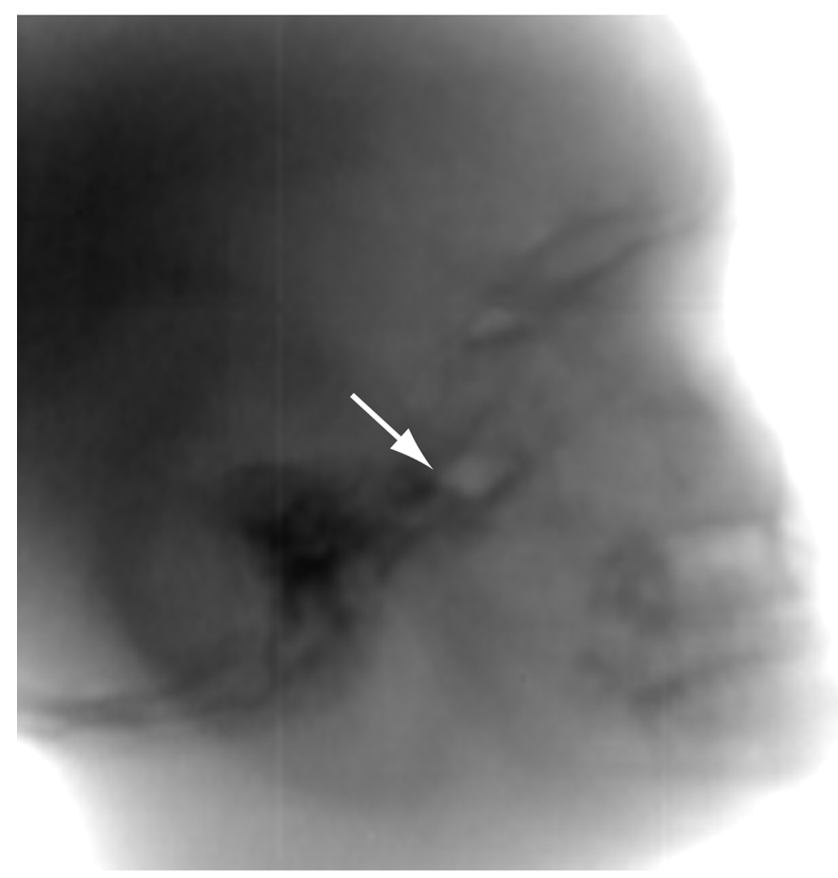

(a)

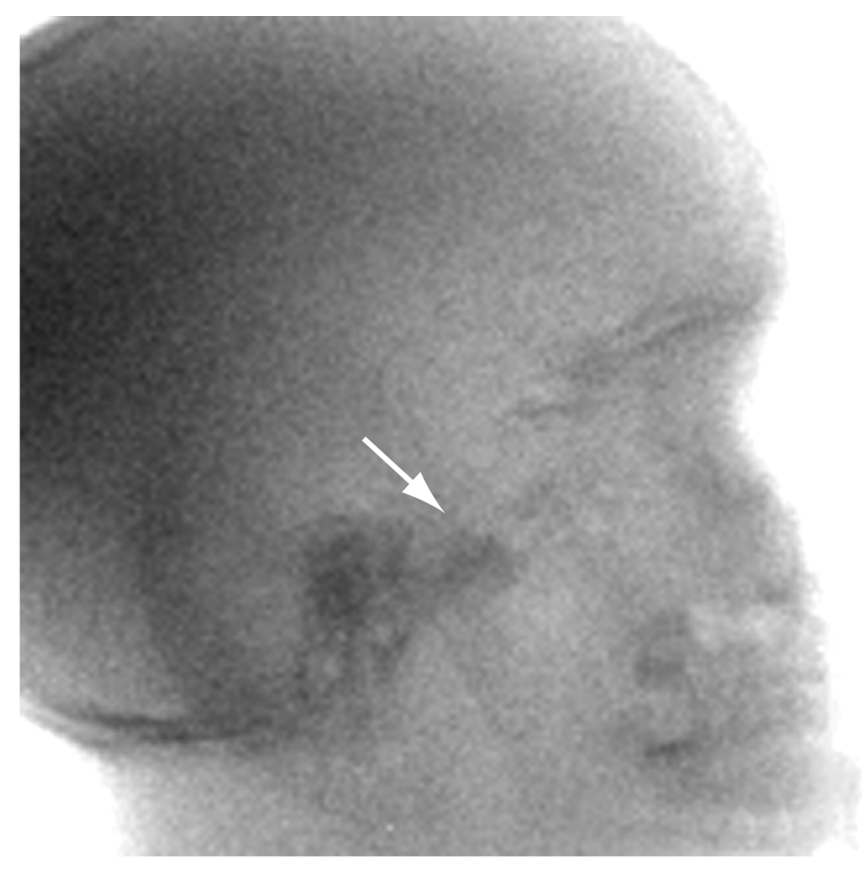

(b)

FIG. 9. Images of a humanoid skull phantom acquired at $0.054 \mathrm{MU}$ using (a) the prototype detector incorporating the white top layer and a $1 \mathrm{~mm}$ thick Cu plate (b) a conventional EPID. The arrows indicate the location of a low-contrast feature, and are included in order to facilitate comparison between the two images.

(such as the one indicated by the arrow) compared to the image obtained from the conventional system.

\section{DISCUSSION AND CONCLUSION}

One of the most desirable goals of modern image-guided radiotherapy is to clearly delineate the clinical target volume (i.e., the visible tumor volume along with an estimated margin to include microscopic extensions of the tumor) from surrounding normal tissue, with the patient in the treatment position, immediately prior to, and, even during dose delivery. ${ }^{1}$ Such capability would allow the implementation of increasingly conformal treatment plans, while avoiding errors due to intrafraction and interfraction organ motion with respect to surrogates such as bony landmarks. The realization of this goal would be significantly aided by imaging modalities capable of resolving soft-tissue contrast at clinically acceptable doses, along with the seamless integration of such modalities within the treatment setup. In this work, we have demonstrated that high-DQE megavoltage imagers based on thick, segmented, crystalline detectors show great potential for low-dose soft-tissue contrast resolution from a "beam's eye view." As seen from the results, the prototype segmented crystal-based imager is capable of resolving low-contrast objects using extremely small amounts of radiation-a consequence of its order of magnitude higher DQE compared to that of current commercially available EPIDs.

The ability of the prototype system to acquire high quality images using only a few beam pulses has important implications for projection as well as tomographic imaging. In the case of projection imaging, such an EPID could perform image-guided localization and verification using over an order of magnitude less radiation (0.027 to $0.081 \mathrm{MU})$ than currently used ( 3 to $4 \mathrm{MU}$ per image at our institution). It would also become possible to acquire a sequence of image frames in fluoroscopic mode, using only one or two linac pulses per frame, in order to record and, potentially, correct for patient movement during treatment.

In the case of megavoltage tomographic imaging, where current EPIDs face a severe tradeoff between dose and the contrast to noise ratio (CNR), defined as the ratio of the image contrast to the voxel noise, ${ }^{8,20}$ an increase in DQE can lead to a proportional reduction in dose. This is illustrated by the following relationship, derived by Barrett et al. ${ }^{63}$ between the voxel noise $\sigma_{\mathrm{vox}}$ in a reconstructed slice, the dose and the DQE

$$
\sigma_{\mathrm{vox}}^{2} \propto \frac{1}{\eta D_{\text {center }}},
$$

where $D_{\text {center }}$ is the dose to the center of the phantom and $\eta$ is interpreted as the zero frequency DQE of the system. ${ }^{31}$ Thus, in order to achieve the same CNR as a conventional, phosphor screen-based EPID (DQE $[0] \approx 1 \%)$, the prototype system $(\mathrm{DQE}[0] \approx 22 \%)$, would ideally require only $\sim 1 / 22$ times the dose. Therefore, the present DQE results strongly suggest that MVCT could be performed using a high-DQE system such as the prototype imager at clinically acceptable doses. For example, a set of 100 MVCT projections, each using a single pulse $(0.027 \mathrm{MU})$, could be acquired using comparable, or, even less radiation (2.7 MU) than that used by current commercial EPIDs to acquire a single projection 
image ( 3 to $4 \mathrm{MU}$ ). Equally important is the fact that the corresponding dose $(\sim 2 \mathrm{cGy}$ at the isocenter, as explained in Sec. II B.) would be comparable to those encountered in kilovoltage cone-beam CT scans. ${ }^{64}$

Notwithstanding these highly promising possibilities, it can be seen from the results that in order to incorporate a segmented detector based on the prototype examined in the present study into a clinical EPID, a number of practical issues will have to be addressed. First, fabrication techniques need to be refined in order to achieve uniform center-tocenter spacing between the scintillator elements in both directions-a requirement for accurate registration of the scintillator elements to the underlying array pixels. Such improvements are required in two areas-achieving pitch uniformity within a single segmented detector module and achieving uniformity between adjacent modules. In order to achieve uniformity of element pitch along both directions within a single module, the precision with which the segmented elements are formed needs to be improved. Moreover, it is necessary to ensure that the thickness and the uniformity of the boundaries of each small-area module are carefully controlled so that the element pitch is maintained when the individual blocks are joined together to form a large-area detector. Finally, improvements are required in the techniques used to perform the "block-to-block" alignment so as to maintain the element pitch across the entire imaging area of the detector along both directions. As fabrication techniques improve, it is expected that higher spatial resolution can be achieved through the creation of segmented detectors with finer and highly regular element pitch so that each segmented detector element is registered to a single pixel of the underlying active matrix array such as the $508 \mu \mathrm{m}$ pitch array used in these studies.

Second, the optical properties of the septal walls need to be optimized so as to minimize DQE loss due to the Lubberts effect and Swank noise. As explained in Sec. III E, the loss in DQE due to the optical component of the Lubberts effect can be minimized by fabricating highly opaque septal walls. The DQE degradation due to optical Swank noise (as quantified by the optical Swank factor, $\approx 0.8$ ) is relatively small in the present prototype, considering the $40 \mathrm{~mm}$ long optical path. By comparison, the optical Swank factor for a $2 \mathrm{~mm}$ thick powdered phosphor-based detector is estimated to be $\sim 0.2{ }^{37}$ However, it is likely that, for segmented detector elements with higher aspect ratios (i.e., taller cells and/or finer pitch), there will be greater depth dependence in the optical gain due to increased absorption of the optical photons along the septa, thereby resulting in higher levels of Swank noise. In order to minimize the effect of this noise source, septal walls with very high reflectivity and scintillator materials which exhibit very high light transmission, such as bismuth germanate (BGO), ${ }^{65,66}$ could be incorporated. The use of such materials would ensure that most of the light generated at the x-ray side of the detector reaches the underlying active matrix array. Another, more complex strategy would involve the fabrication of septal walls with "graded reflectivity." Such septal walls would be more reflective nearer to the top, i.e., the x-ray side, of the detector compared to the bottom, i.e., the array side. Such an arrangement, possibly in combination with highly transparent scintillators such as BGO, could also reduce the depth dependence of the optical gain.

In the case of thick (greater than $\sim 10 \mathrm{~mm}$ ), large-area $\left(\sim 40 \times 40 \mathrm{~cm}^{2}\right)$ segmented detectors, there is likely to be significant loss of MTF and consequent DQE degradation at the peripheral regions of the detector due to the diverging trajectories of x-rays from the source. Different strategies can be used to mitigate this effect, depending upon the imaging requirements. For example, the segmented detector elements could be fabricated so that they are "focused" toward the x-ray source. ${ }^{35}$ Ideally, such a focused detector would not exhibit any loss in DQE at the periphery due to off-axis $x$ rays, independent of detector thickness. Of course, it is likely that more sophisticated fabrication techniques will be needed in order to create such focused detectors. An alternative approach would be to reduce the detector thickness by making use of higher-density scintillator and septal wall materials in order to achieve DQE performance comparable to a thicker, lower-density configuration. For example, theoretical calculations indicate that $\mathrm{DQE}$ performance equal or superior to that of the prototype system, which is based on a $40 \mathrm{~mm}$ thick, CsI(Tl) detector with polystyrene septa, may be achieved using a $10 \mathrm{~mm}$ thick, BGO detector incorporating tungsten septa. 35

Finally, it is anticipated that, with further optimization of the segmented detector design, involving the incorporation of thick, higher-density scintillator and septal wall materials such as BGO and tungsten, respectively, it may be possible to achieve further increases in the DQE up to $\sim 50 \% .{ }^{35}$ The relationship shown in Eq. (5) suggests that, for such configurations, the CNR for tomographic images will correspondingly improve, potentially enabling even better resolution of soft-tissue contrast at low doses.

\section{ACKNOWLEDGMENTS}

The authors would like to thank Mike Mayhugh and Peter Menge, St. Gobain Crystals, Ohio, for their support and guidance during the design of the prototype detector. We would like to express our appreciation to Jeff Siewerdsen, Princess Margaret Hospital, Toronto, Canada, for helpful discussions on CT imaging performance. This work was supported by Grant No. RO1 CA51397 from the National Institutes of Health.

\footnotetext{
a)Elecronic mail: asawant@umich.edu

${ }^{1}$ T. R. Mackie et al., "Image guidance for precise conformal radiotherapy," Int. J. Radiat. Oncol., Biol., Phys. 56, 89-105 (2003).

${ }^{2}$ J. L. Barker et al., "Quantification of volumetric and geometric changes occuring during fractionated radiotherapy for head-and-neck cancer using an integrated CT/linear accelerator system," Int. J. Radiat. Oncol., Biol., Phys. 59, 960-970 (2004).

${ }^{3} \mathrm{~K}$. Kuriyama et al., "A new irradiation unit constructed of self-moving gantry-CT and linac,” Int. J. Radiat. Oncol., Biol., Phys. 55, 428-435 (2003).

${ }^{4} \mathrm{~J}$. M. Balter et al., "Daily targeting of intrahepatic tumors for radiotherapy,” Int. J. Radiat. Oncol., Biol., Phys. 52, 266-271 (2002).

${ }^{5}$ D. A. Jaffray et al., "Dual-beam imaging for online verification of radio-
} 
therapy field placement," Int. J. Radiat. Oncol., Biol., Phys. 33, $1273-$ 1280 (1995).

${ }^{6}$ D. A. Jaffray et al., "A radiographic and tomographic imaging system integrated into a medical linear accelerator for localization of bone and soft-tissue targets," Int. J. Radiat. Oncol., Biol., Phys. 45, 773-789 (1999).

${ }^{7}$ D. A. Jaffray et al., "Flat-panel cone-beam computed tomography for image-guided radiation therapy," Int. J. Radiat. Oncol., Biol., Phys. 53, 1337-1349 (2002).

${ }^{8}$ B. A. Groh et al., "A performance comparison of flat-panel imager-based MV and kV cone-beam CT," Med. Phys. 29, 967-975 (2002).

${ }^{9}$ J. Lattanzi et al., "Ultrasound-based stereotactic guidance of precision conformal external beam radiation therapy in clinically localized prostate cancer," Urology 55, 73-78 (2000).

${ }^{10} \mathrm{~K}$. M. Langen et al., "Evaluation of ultrasound-based prostate localization for image-guided radiotherapy," Int. J. Radiat. Oncol., Biol., Phys. 57, 635-644 (2003).

${ }^{11}$ S. L. Meeks et al., "Ultrasound-guided extracranial radiosurgery: Technique and application,” Int. J. Radiat. Oncol., Biol., Phys. 55, 1092-1101 (2003).

${ }^{12}$ K. M. Langen and D. T. Jones, "Organ motion and its management," Int. J. Radiat. Oncol., Biol., Phys. 50, 265-278 (2001).

${ }^{13}$ C. G. Rowbottom and D. A. Jaffray, "Development of an integral system test for image-guided radiotherapy," Med. Phys. 31, 3500-3505 (2004).

${ }^{14}$ R. G. Simpson et al., "A 4-MV CT scanner for radiation therapy: The prototype system," Med. Phys. 9, 574-579 (1982).

${ }^{15}$ W. Swindell et al., "Computed tomography with a linear accelerator with radiotherapy applications,” Med. Phys. 10, 416-420 (1983).

${ }^{16}$ F. A. Ghelmansarai et al., "Soft tissue visualization using a highly efficient megavoltage cone beam CT imaging system," Proc. SPIE 5745, 159-170 (2005)

${ }^{17}$ E. J. Seppi et al., "Megavoltage cone-beam computed tomography using a high-efficiency image receptor," Int. J. Radiat. Oncol., Biol., Phys. 55, 793-803 (2003).

${ }^{18} \mathrm{~J}$. Pouliot et al., "Low-dose megavoltage cone-beam CT for radiation therapy," Phys. Med. Biol. 61, 552-560 (2005).

${ }^{19}$ E. Ford et al., "Low-dose megavoltage cone-beam CT with an amorphous silicon electronic portal imaging device," Med. Phys. 29, 1241-1241 (2002).

${ }^{20}$ E. C. Ford et al., "Cone-beam CT with megavoltage beams and an amorphous silicon electronic portal imaging device: Potential for verification of radiotherapy of lung cancer," Med. Phys. 29, 2913-2924 (2002).

${ }^{21}$ H. Guan, "Megavoltage portal CT using an amorphous silicon imaging device-with comparison to a CCD based EPID system," Med. Phys. 29, 1243-1243 (2002).

${ }^{22}$ H. Q. Guan and Y. P. Zhu, "Feasibility of megavoltage portal CT using an electronic portal imaging device (EPID) and a multi-level scheme algebraic reconstruction technique (MLS-ART)," Phys. Med. Biol. 43, 29252937 (1998).

${ }^{23}$ M. A. Mosleh-Shirazi, W. Swindell, and P. M. Evans, "Optimization of the Scintillation Detector in a Combined 3D Megavoltage CT Scanner and Portal Imager," Med. Phys. 25, 1880-1890 (1998).

${ }^{24}$ T. T. Monajemi et al., "Modeling scintillator-photodiodes as detectors for megavoltage CT," Med. Phys. 31, 1225-1234 (2004).

${ }^{25} \mathrm{H}$. Keller et al., "Monte Carlo study of a highly efficient gas ionization detector for megavoltage imaging and image-guided radiotherapy," Med. Phys. 29, 165-175 (2002).

${ }^{26}$ K. J. Ruchala et al., "Megavoltage CT on a Tomotherapy System," Phys. Med. Biol. 44, 2597-2621 (1999).

${ }^{27}$ A. Brahme, "Design principles and clinical possibilities with a new generation of radiation therapy equipment," Acta Oncol. 26, 403-412 (1987).

${ }^{28}$ K. M. Langen et al., "The use of megavoltage CT (MVCT) images for dose recomputations," Phys. Med. Biol. 50, 4259-4276 (2005).

${ }^{29}$ H. E. Johns and J. R. Cunningham, The Physics of Radiology, 4th ed. (Charles C. Thomas, Springfield, IL, 1983).

${ }^{30} \mathrm{M}$. Endo et al., "Effect of scattered radiation on image noise in cone beam CT," Med. Phys. 28, 469-474 (2001).

${ }^{31}$ J. H. Siewerdsen and D. A. Jaffray, "Cone-beam computed tomography with a flat-panel imager: Magnitude and effects of x-ray scatter," Med. Phys. 28, 220-231 (2001).

${ }^{32}$ G. H. Glover and N. J. Pelc, "An algorithm for the reduction of metal clip artifacts in CT reconstructions," Med. Phys. 8, 799-807 (1981).
${ }^{33}$ E. K. Fishman et al., "Metallic hip implants: CT with multiplanar reconstruction," Radiology 160, 675-681 (1986).

${ }^{34}$ J. Sillanpaa et al., "Developments in megavoltage cone beam CT with an amorphous silicon EPID: reduction of exposure and synchronization with respiratory gating," Med. Phys. 32, 819-829 (2005).

${ }^{35}$ A. Sawant et al., "Segmented crystalline scintillators: An initial investigation of high quantum efficiency detectors for megavoltage x-ray imaging," Med. Phys. 32, 3067-3083 (2005).

${ }^{36}$ M. A. Mosleh-Shirazi et al., "Rapid portal imaging with a high-efficiency, large field-of-view detector," Med. Phys. 25, 2333-2346 (1998).

${ }^{37}$ A. Sawant et al., "Segmented phosphors: MEMS-based high quantum efficiency detectors for megavoltage x-ray imaging," Med. Phys. 32, $553-565$ (2005).

${ }^{38} \mathrm{~A}$. Sawant et al., "Theoretical analysis and experimental evaluation of a CsI(Tl) based electronic portal imaging system," Med. Phys. 29, 10421053 (2002).

${ }^{39}$ L. E. Antonuk et al., "Initial performance evaluation of an indirectdetection, active matrix flat-panel imager (AMFPI) prototype for megavoltage imaging," Int. J. Radiat. Oncol., Biol., Phys. 42, 437-454 (1998).

${ }^{40}$ L. E. Antonuk, "Electronic portal imaging devices: A review and historical perspective of contemporary technologies and research," Phys. Med. Biol. 47, R31-R65 (2002).

${ }^{41}$ L. E. Antonuk et al., "Megavoltage imaging with a large area, flat-panel, amorphous silicon imager," Int. J. Radiat. Oncol., Biol., Phys. 36(3), 661-672 (1996).

${ }^{42}$ L. E. Antonuk et al., "Empirical investigation of the signal performance of a high-resolution, indirect detection, active matrix flat-panel imager (AMFPI) for fluoroscopic and radiographic operation," Med. Phys. 24, 51-70 (1997).

${ }^{43}$ Y. El-Mohri et al., "Determination of the detective quantum efficiency of a prototype, megavoltage indirect detection, active matrix flat-panel imager," Med. Phys. 28, 2538-2550 (2001).

${ }^{44}$ W. Huang et al., "An asynchronous, pipelined, electronic acquisition system for active matrix flat-panel imagers (AMFPIs)," Nucl. Instrum. Methods Phys. Res. A 431, 273-284 (1999).

${ }^{45} \mathrm{H}$. Fujita et al., "A simple method for determining the modulation transfer-function in digital radiography," IEEE Trans. Med. Imaging 11, 34-39 (1992).

${ }^{46}$ J. T. Dobbins, "Image quality metrics for digital systems," in Handbook of Medical Imaging, edited by J. Beutel, H. L. Kundel, and R. L. Van Metter (SPIE Press, Bellingham, Washington, 2000), pp. 163-222.

${ }^{47} \mathrm{P}$. Munro and D. C. Bouius, "X-ray quantum limited portal imaging using amorphous silicon flat-panel arrays," Med. Phys. 25, 689-702 (1998).

${ }^{48} \mathrm{~F}$. Cremers et al., "Performance of electronic portal imaging devices (EPIDs) used in radiotherapy: Image quality and dose measurements," Med. Phys. 31, 985-996 (2004).

${ }^{49}$ J. H. Siewerdsen et al., "Signal, noise power spectrum, and detective quantum efficiency of indirect-detection flat-panel imagers for diagnostic radiology," Med. Phys. 25, 614-628 (1998).

${ }^{50} \mathrm{P}$. R. Granfors and R. Aufrichtig, "DQE(f) of an amorphous-silicon flatpanel $\mathrm{x}$-ray detector: Detector parameter influences and measurement methodology," Proc. SPIE 3977, 2-13 (2000).

${ }^{51} \mathrm{P}$. R. Granfors et al., "Performance of a $41 \times 41 \mathrm{~cm}^{2}$ amorphous silicon flat panel $\mathrm{x}$-ray detector designed for angiographic and R\&F imaging applications," Med. Phys. 30, 2715-2726 (2003).

${ }^{52}$ D. W. O. Rogers, "Fluence to dose equivalent conversion factors calculated with EGS3 for electrons from 100-Kev to 20-Gev and Photons from 11-Kev to 20-Gev," Health Phys. 46, 891-914 (1984).

${ }^{53} \mathrm{D}$. Sheikh-Bagheri, "Monte carlo study of photon beams from medical linear accelerators: Optimization, benchmark and spectra," $\mathrm{Ph}$. D. thesis, Dept. of Physics, Carleton University, 1999.

${ }^{54} \mathrm{G}$. Lubberts, "Random noise produced by x-ray fluorescent screens," J. Opt. Soc. Am. 58, 1475-1483 (1968).

${ }^{55}$ R. M. Nishikawa, M. J. Yaffe, and R. B. Holmes, "Effect of finite phosphor thickness on detective quantum efficiency," Med. Phys. 16, 773 780 (1989).

${ }^{56}$ J. A. Treurniet, B. R. B. Walters, and D. W. O. Rogers, "BEAMnrc, DOSXYZnrc and BEAMDP GUI User's Manual," NRC Report PIRS 0623(rev B) (2001).

${ }^{57}$ I. Kawrakow and D. W. O. Rogers, "The EGSnrc code system: Monte Carlo simulation of electron and photon transport," Technical Report PIRS-701, National Research Council of Canada, Ottawa, Canada, 2000.

${ }^{58}$ C. Kirkby and R. Sloboda, "Comprehensive Monte Carlo calculation of 
the point spread function for a commercial a-Si EPID," Med. Phys. 32, 1115-1127 (2005).

${ }^{59}$ D. A. Jaffray et al., "Monte-Carlo studies of x-ray-energy absorption and quantum-noise in megavoltage transmission radiography," Med. Phys. 22, 1077-1088 (1995).

${ }^{60}$ R. K. Swank, “Absorption and noise in x-ray phosphors," J. Appl. Phys. 44, 4199-4203 (1973).

${ }^{61}$ J. Rowlands and J. Yorkston, "Flat panel detectors for digital radiography," in Handbook of Medical Imaging, edited by J. Beutel, H. L. Kundel, and R. L. Van Metter (SPIE Press, Bellingham, Washington, 2000), pp. 223-328.

${ }^{62}$ W. Viehmann, J. F. Arens, and M. Simon, "Optical transmission measurements on monocrystalline and polycrystalline cesium iodide," Nucl. In- strum. Methods 116, 283-295 (1974).

${ }^{63}$ H. H. Barrett, S. K. Gordon, and R. S. Hershel, "Statistical limitations in transaxial tomography," Comput. Biol. Med. 6, 307-323 (1976).

${ }^{64}$ D. Letourneau et al., "Cone-beam-CT guided radiation therapy: Technical implementation," Radiol. Oncol. 75, 279-286 (2005).

${ }^{65}$ D. J. Krus, W. P. Novak, and L. Perna, "Precision linear and twodimensional scintillation crystal arrays for $\mathrm{x}$-ray and gamma-ray imaging applications," Proceedings of SPIE on Hard X-Ray, Gamma-Ray, and Neutron Detector Physics, 3768, 183-194 (1999).

${ }^{66} \mathrm{P}$. Kozma and P. Kozma, "Radiation resistivity of BGO crystals due to low-energy gamma-rays," Nucl. Instrum. Methods Phys. Res. A 501, pp. 499-504 (2003). 